\title{
Trend of Non-contrast Chest Computed Tomography Use in the Lung Cancer Screening Era: SEER-Medicare 2008-2016
}

J Gen Intern Med 36(11):3589-91

DOI: $10.1007 / \mathrm{s} 11606-020-06586-4$

(c) Society of General Internal Medicine 2021

\section{INTRODUCTION}

After the National Lung Screening Trial (NLST) demonstrated the efficacy of low-dose computed tomography (LDCT) screening in August 2011, ${ }^{1}$ the U.S. Preventive Services Task Force (USPSTF) recommended this procedure in December 2013 (Grade B). ${ }^{2}$ Under the Affordable Care Act, insurers were required to cover LDCT starting in January 2014, yet the Centers for Medicare \& Medicaid Services (CMS) did not reimburse LDCT until February $2015 .{ }^{3}$ To date, little is known about how dissemination of evidence, guidelines, and reimbursement policy affected physicians' practice. We hypothesized that physicians ordered non-contrast chest CT (NCCCT) instead of LDCT before the Common Procedure Terminology (CPT) code of LDCT became available in 2015. We also anticipated this would change after Medicare reimbursed LDCT.

\section{METHODS}

Analyzing the 5\% non-cancer sample from SEER-Medicare data, we identified Medicare fee-for-service beneficiaries aged 65-77 on January 1 of each year, 2008-2016. We calculated the seasonal percentage of beneficiaries who received NCCCT and LDCT. We calculated non-contrast abdominal CT use for comparison. We reviewed the principal diagnosis for each NCCCT claim.

\section{RESULTS}

Approximately $0.71 \%$ beneficiaries underwent NCCCTs in January-March 2008. The utilization remained stable after the dissemination of NLST, at $0.73 \%$ in October-December 2013 (Fig. 1). After the USPSTF guidelines were published (December 2013), the percentage rose, reaching $0.91 \%$ in January-March 2015 when the CMS started reimbursing

Received July 26, 2020

Accepted December 30, 2020

Published online January 19, 2021
LDCT and $1.06 \%$ in October-December 2016. LDCT claims did not occur until January-March 2015 and reached $0.09 \%$ in October-December 2016. We estimated that approximately $8 \%$ of the beneficiaries undergoing NCCCT might have transitioned to LDCT. In comparison, non-contrast abdominal CT use only increased from $0.95 \%$ in October-December 2013 to $1.12 \%$ in October-December 2016. Over the 3-year period, non-contrast abdominal CT use increased $18.1 \%$ while NCCCT use increased $44.3 \%(P=0.004)$. The analysis of percentages of principal diagnoses for NCCCT claims revealed an increase in the code of "solitary pulmonary nodule," from $11.7 \%$ (918/7844 claims) in 2013 to $23.4 \%$ (3003/12,832 claims) in 2016 (Fig. 2).

\section{DISCUSSION}

After the USPSTF guidelines, NCCCT use increased substantially. The diagnostic code of solitary pulmonary nodule also increased during the same period. Collectively, these findings suggest that physicians may have ordered NCCCT for screening before the LDCT CPT code was developed. The CMS did not reimburse LDCT until 2015, yet national surveys found that 3.3-3.9\% of eligible Americans self-reported receiving such testing between 2010 and $2015 .{ }^{4}$ While the population is different from ours, it is likely that physicians ordered NCCCT for lung cancer screening, or ordered LDCT using NCCCT CPT code. Our study raised concerns about the legitimacy of using other diagnostic codes for screening.

Contradictory to our hypothesis, NCCCT use continued increasing after 2015. Because the CMS requires shared decision-making and smoking cessation counselling concomitant with LDCT screening, it is possible that physicians ordered NCCCT without engaging these processes. Additionally, physicians might use NCCCT for beneficiaries who did not meet the LDCT eligibility criteria. Nevertheless, NCCCT use continued increasing after the CMS reimbursed LDCT, suggesting that physicians inappropriately used NCCCT for screening.

Our findings, limited to Medicare beneficiaries aged 65-77 in the SEER regions, cannot be generalized to people aged 5564 or those who resided in non-SEER regions. Additionally, smoking $\geq 30$ pack-years is a criterion for LDCT screening, but this information was unavailable in our data set, which is a limitation of the study. It remains unclear the impact on current 


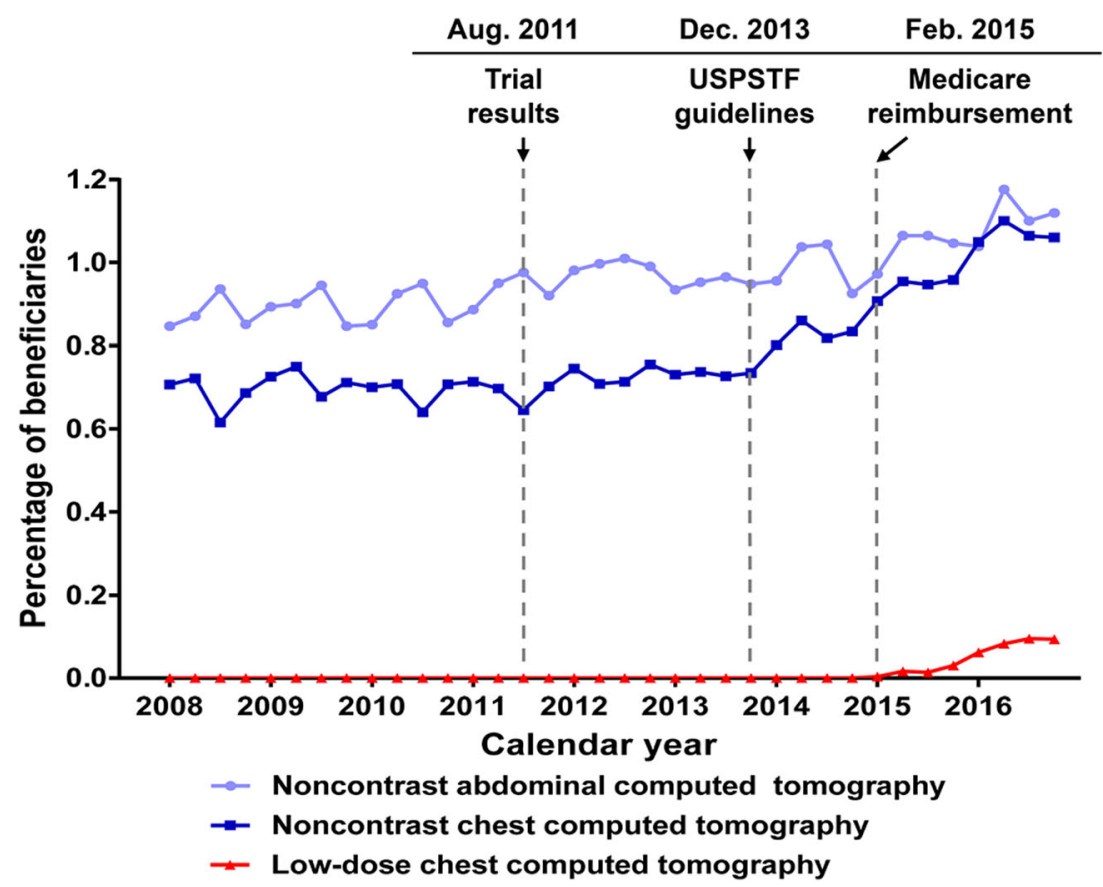

Figure 1 Trends of the use of non-contrast chest and abdominal computed tomography, and low-dose chest computed tomography, 2008-2016. We used Common Procedure Terminology (CPT) codes of 74150 and 74176 to capture all non-contrast abdominal CTs during the study period. We used CPT code of 71250 for non-contrast chest CT, and CPT codes of S8032 and G0297 for LDCT. We used simple linear regressions for slopes of non-contrast abdominal CT use and non-contrast chest CT use between October-December 2013 and OctoberDecember 2016. Significant difference $(P=0.004)$ between two slopes using $F$ test was found. USPSTF, U.S. Preventive Services Task Force.

results if the study population could be restricted to patients who meet the eligibility criteria. We are aware that in late
2011, there was a new diagnosis code of solitary pulmonary nodule, leading to an increase in this diagnosis in 2012.

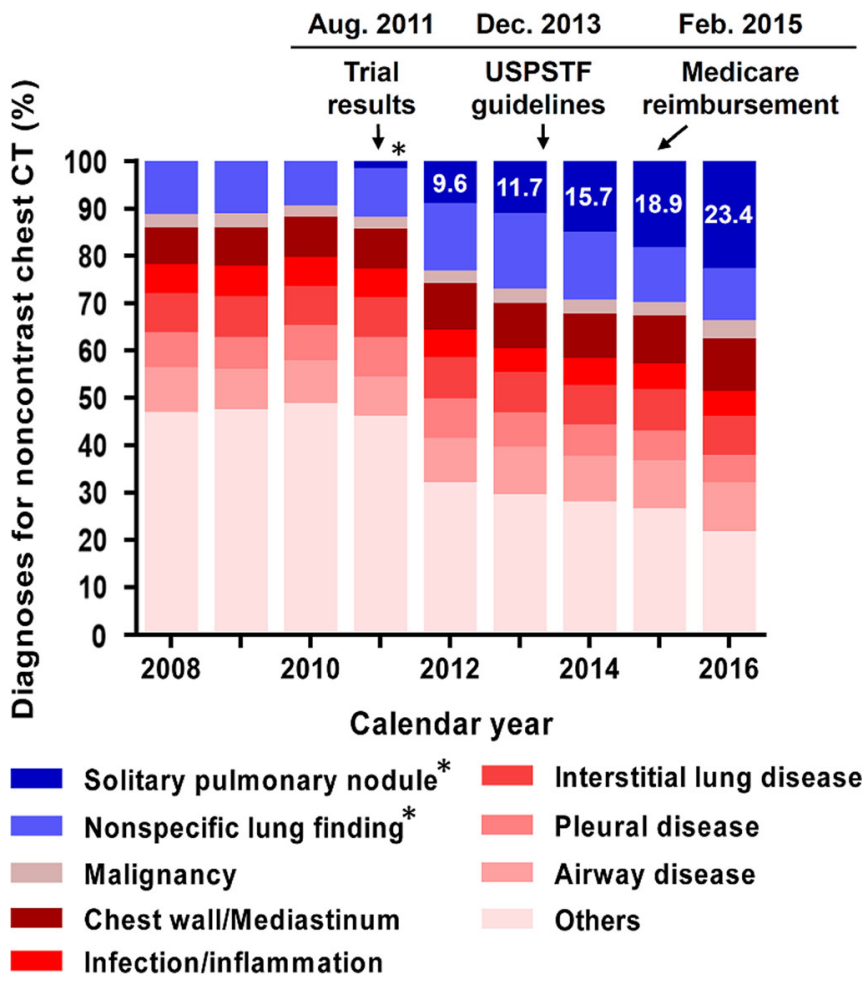

Figure 2 Percentages of the principal diagnosis codes for non-contrast chest computed tomography (CT) by year. The number in each bar denotes the percentage of solitary pulmonary nodule as principal diagnosis. USPSTF, U.S. Preventive Services Task Force. *In late 2011, the International Classification of Diseases, Ninth Revision (ICD-9) code for nonspecific lung finding (793.1) was categorized into solitary pulmonary nodule (ICD-9: 793.11, corresponding to ICD-10: R91.1) and other nonspecific lung finding (ICD-9: 793.19, corresponding to ICD-10: R91.8). Researchers could contact the first author for the detailed $I C D-9 / I C D-10$ codes. 
However, the continuous increase of this diagnosis during 2013-2016 and the parallel increase in NCCCT use support the hypothesis that physicians ordered NCCCT for screening.

In summary, guidelines dissemination was associated with an increase in NCCCT use. The continuous increase despite the reimbursement of LDCT after 2015 raises the possibility that higher dose NCCCT may be misused for screening. The LDCT should be interpreted using the Lung CT Screening Reporting and Data System (Lung-RADS) which has been shown to reduce false-positive results. ${ }^{5}$ As LDCT is the only recommended test for screening, there is a need for collaboration between radiologists and ordering physicians to wisely use LDCT screening. ${ }^{6}$

Acknowledgments: The collection of the California cancer incidence data used in this study was supported by the California Department of Public Health as part of the statewide cancer reporting program mandated by California Health and Safety Code Section 103885; the National Cancer Institute's Surveillance, Epidemiology and End Results Program under contract NO1-PC-35136 awarded to the Northern California Cancer Center, contract NO1-PC-35139 awarded to the University of Southern California, and contract NO2-PC-15105 awarded to the Public Health Institute; and the Centers for Disease Control and Prevention's National Program of Cancer Registries, under agreement \#U55/CCR921930-02 awarded to the Public Health Institute. The authors of this report are responsible for its content. The ideas and opinions expressed herein are those of the authors and endorsement by the State of California, Department of Public Health, the National Cancer Institute, and the Centers for Disease Control and Prevention or their Contractors and Subcontractors is not intended nor should be inferred. The authors acknowledge the efforts of the Applied Research Program, NCI; the Office of Research, Development and Information, CMS; Information Management Services (IMS), Inc.; and the Surveillance, Epidemiology, and End Results (SEER) Program tumor registries in the creation of the SEER-Medicare database. The interpretation and reporting of the SEER-Medicare data are the sole responsibility of the authors.

Szu-Chun Yang, MD, $P h D^{1,2}$

Cary Gross, $M D^{3}$

Jung-Der Wang, $M D, S c D^{2,4}$

Shi-Yi Wang, MD, $P h D^{1,3}$

${ }^{1}$ Department of Chronic Disease Epidemiology, Yale School of Public Health,

New Haven, CT, USA

${ }^{2}$ Department of Internal Medicine, National Cheng Kung University Hospital, College of Medicine, National Cheng Kung University,

Tainan, Taiwan

${ }^{3}$ Cancer Outcomes, Public Policy, and Effectiveness Research Center, Yale School of Medicine,

New Haven, CT, USA
${ }^{4}$ Department of Public Health, College of Medicine, National Cheng Kung University,

Tainan, Taiwan

Corresponding Author: Shi-Yi Wang, MD, PhD; Department of Chronic Disease Epidemiology, Yale School of Public Health, New Haven, CT, USA (e-mail: shiyi.wang@yale.edu).

Authors' Contribution All authors had access to the data and a role in writing the manuscript.

Funding Dr. Yang reports grants from the Ministry of Science and Technology (MOST108-2918-I-006-006) and National Cheng Kung University Hospital (NCKUH-11002029) during the conduct of the study. Dr. Gross reports grants from Johnson \& Johnson, NCCN Foundation (Pfizer/AstraZeneca), Flatiron Health, and Genentech outside the submitted work. Dr. S.Y. Wang reports grants from Genentech outside the submitted work. No other disclosures were reported.

\section{Compliance with Ethical Standards:}

Conflict of Interest: The authors declare that they do not have a conflict of interest.

\section{REFERENCES}

1. Aberle DR, Adams AM, Berg CD, Black WC, Clapp JD, Fagerstrom RM, et al. Reduced lung-cancer mortality with low-dose computed tomographic screening. N Engl J Med. 2011; 365(5): 395-409. https://doi.org/10. 1056/NEJMoa1102873.

2. Moyer VA. Screening for lung cancer: U.S. Preventive Services Task Force recommendation statement. Ann Intern Med. 2014; 160(5): 330-8. https://doi.org/10.7326/m13-2771.

3. Centers for Medicare \& Medicaid Services. Medicare coverage of screening for lung cancer with low dose computed tomography (LDCT). https://www. cms.gov/Outreach-and-Education/Medicare-Learning-Network-MLN/ MLNMattersArticles/Downloads/MM9246.pdf. Assessed February 2, 2020.

4. Jemal A, Fedewa SA. Lung cancer screening with low-dose computed tomography in the United States-2010 to 2015. JAMA Oncol. 2017; 3(9): 1278-81. https://doi.org/10.1001/jamaoncol.2016.6416.

5. Pinsky PF, Gierada DS, Black W, Munden R, Nath H, Aberle D, et al. Performance of Lung-RADS in the National Lung Screening Trial: a retrospective assessment. Ann Intern Med. 2015; 162(7): 485-91. https://doi.org/10.7326/m14-2086.

6. The American College of Radiology, Radiological Society of North America, American Society of Radiological Technologists and American Association of Physicists in Medicine. 2020 Image Wisely: Pledge for Referring Practitioners. Available at: https://www.imagewisely.org/Pledge/Referring-Practitioner. Accessed November 16, 2020.

Publisher's Note: Springer Nature remains neutral with regard to jurisdictional claims in published maps and institutional affiliations. 